\title{
Fuzzy Pattern Trees as an Alternative to Rule-based Fuzzy Systems: Knowledge-driven, Data-driven and Hybrid Modeling of Color Yield in Polyester Dyeing
}

\author{
Maryam Nasiri \\ Software Engineering Institute \\ University of Siegen \\ Siegen, Germany \\ nasiri@informatik.uni-siegen.de
}

\author{
Thomas Fober, Robin Senge, Eyke Hüllermeier \\ Department of Mathematics and Computer Sciences \\ University of Marburg \\ Marburg, Germany \\ \{thomas, senge, eyke\}@mathematik.uni-marburg.de
}

\begin{abstract}
This paper advocates a novel approach to fuzzy systems modeling called fuzzy pattern trees. This approach is largely motivated by alleged disadvantages of rule-based system architectures that still dominate the field. Due to its hierarchical, modular structure and the use of different types of (nonlinear) aggregation operators, a fuzzy pattern tree has the ability to represent functional dependencies in a more flexible and more compact way, thereby offering a reasonable balance between accuracy and model transparency. We evaluate this new model class in the context of a concrete case study, namely the modeling of color yield in polyester high temperature dyeing as a function of disperse dyes concentration, temperature and time. To this end, we compare three possibilities for model construction: purely knowledge-driven, purely data-driven and a hybrid approach combining these two. Our results show that, in comparison to conventional fuzzy modeling using Mamdani rules, fuzzy pattern trees are not only more accurate but also more compact and therefore more easily interpretable, regardless of whether the models are constructed in a knowledge-driven, data-driven or hybrid manner. Moreover, we show that a hybrid modeling approach can outperform a purely data-driven and a purely knowledge-driven approach if expert knowledge and model calibration are combined in a suitable way.
\end{abstract}

\section{INTRODUCTION}

The transparency and interpretability of fuzzy systems is often emphasized as one of their key advantages, especially in comparison to so-called "black-box" approximation methods such as neural networks. Nowadays, however, models are often extracted from data in an automatic way instead of being designed by a human expert. For this kind of data-driven fuzzy modeling, interpretability becomes a critical issue. For example, a linguistic representation may become difficult if the fuzzy sets have been induced by the learning algorithm in a data-driven way, since the existence of appropriate linguistic interpretations cannot be guaranteed in that case. Another problem that may hamper interpretability concerns the complexity of models consisting of a potentially large number of interacting pieces, for example rules in a rule-based system. Since accurate models typically require a certain level of complexity, accuracy and understandability are to some extent conflicting goals $[1,2]$.

Due to these reasons, the interpretability of fuzzy models is clearly not self-evident and does not come for free, especially when these models are constructed in a data-driven way. Research in this field is still hampered by the lack of accepted criteria for measuring interpretability in a more or less objective way, although some advances have recently been made $[3,4]$. In this paper, we therefore opt for another approach: Instead of looking for generic evaluation measures, we compare different methods in the context of a concrete case study, namely the modeling of color yield in polyester high temperature dyeing.

This paper is a continuation of our previous work [5], in which we compared knowledge-driven and data-driven approaches to fuzzy systems modeling. Here, our goal is to elaborate on possible advantages of a hybrid approach, that is, a combination of knowledge-driven and data-driven modeling. In this regard, we specifically focus on so-called fuzzy pattern trees (FPT) as an alternative approach to fuzzy systems design that has been proposed only recently $[6,7]$. Whereas conventional rule-based fuzzy systems have a "flat" structure, fuzzy pattern trees are hierarchical models. As argued in [8], they are thus able to represent models in more compact way.

Indeed, our results will show that, in comparison to conventional fuzzy modeling using Mamdani rules, fuzzy pattern trees are not only more accurate but also more compact and therefore more easily interpretable, regardless of whether the models are constructed in a knowledge-driven, data-driven or hybrid manner. Moreover, we show that a hybrid modeling approach can outperform a purely data-driven and a purely knowledge-driven approach if both are combined in a suitable way.

The remainder of the paper is organized as follows. In the next section, we briefly recall the basic conception of fuzzy pattern trees. Our application, the modeling of color yield in polyester high temperature dyeing, is outlined in Section 3. The different approaches to model construction are explained in Section 4, and the results of our case study are then presented and discussed in Section 5. The paper ends with some concluding remarks in Section 6.

\section{Fuzzy Pattern Trees}

Pattern tree induction was recently introduced as a novel machine learning method for classification in [6] and further developed for regression in [8]. Roughly speaking, a fuzzy pattern tree is a hierarchical, tree-like structure, whose inner nodes are marked with generalized (fuzzy) logical and arithmetic operators (namely t-norms, t-conorms, average and ordered weighted average operators), and whose leaf nodes are associated with fuzzy predicates on input attributes (fuzzy subsets of the attribute's domain, possibly associated with a linguistic term); see Fig. 1 for an illustration. A pattern tree 


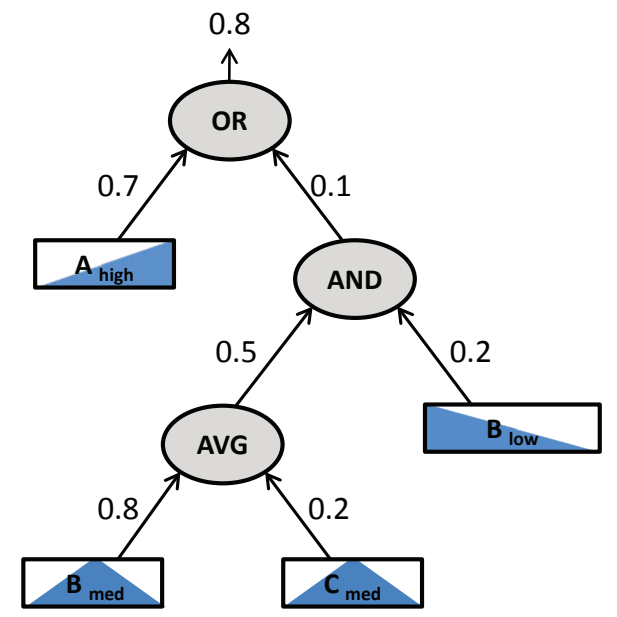

Fig. 1: Example of a fuzzy pattern tree: The output is large if attribute A is high or if the truth degree of a second criterion is high, namely that $\mathrm{B}$ is low and that the average between the conditions that $\mathrm{B}$ and $\mathrm{C}$ are medium is high. The tree also shows the concrete propagation of values from the bottom to the top; in this case, the output is high to the degree 0.8 .

propagates information from the bottom to the top: A node takes the values of its descendants as input, combines them using the respective operator, and submits the output to its predecessor.

Just like other fuzzy models such as Takagi-Sugeno systems [9], a pattern tree can implement a mapping $\mathbb{R}^{p} \rightarrow \mathbb{R}$. Note that the direct output of a pattern tree is in $[0,1]$. One can think of this value as the degree of membership of a fuzzy subset $G$ of an underlying domain $\mathcal{Y} \subseteq \mathbb{R}$. For example, if $\mathcal{Y}$ is an interval $[a, b]$, i.e., if the original output variable is lowerbounded by $a$ and upper-bounded by $b$, then the membership function could be given by a simple linear scaling

$$
G: y \mapsto \frac{y-a}{b-a} .
$$

Thus, the corresponding fuzzy set could be interpreted as a model of the linguistic term "large". Likewise, if the original output is unbounded, a possible re-scaling is

$$
G: y \mapsto \frac{1}{1+\exp (-\alpha y)} .
$$

Considering the fuzzy set $G$ as a fuzzy predicate or, say, property of the output variable (e.g., being large), a fuzzy pattern tree can be seen as a model that specifies criteria on the input attributes which imply this property to hold. From a modeling point of view, the pattern tree approach is based on three important conceptions:

- fuzzification of input attributes;

- hierarchical structuring of a functional dependency through recursive partitioning of criteria into sub-criteria;

- flexible aggregation of sub-criteria by means of parameterized fuzzy operators.

Pattern trees are interesting for several reasons. From a learning point of view, they offer a flexible model class that is able to fit non-linear functions in a quite accurate way, possesses desirable monotonicity properties and can easily handle missing or imprecise input values [7]. Moreover, pattern trees are interesting from an interpretation point of view. A tree can be considered as a kind of generalized logical description of properties that guarantee a "large" output. The description itself is compact and modular due to its hierarchical structure.

Different algorithms have been proposed for learning a pattern tree classifier from a given set of data, namely methods that construct trees in a bottom-up [6] and in a top-down manner [7]. A variant of pattern tree induction suitable for learning regression functions was proposed in [8].

\section{Polyester Dyeing}

The most important man made fiber is Polyethylene terphethalate (PET) commonly known as polyester. This polymer contains ester groups (-CO-O-) in its main molecular chain and is produced by melt spinning process. Ester groups are a result of the reaction between bi-functional carboxylic acids and bi-functional alcohols. The absence of reactive groups, capable of undergoing reaction with anionic and cationic dyes as well as being a hydrophob, has limited dyeing and printing of unmodified polyethylene terphethalate fibers to only disperse dyes. Moreover, under normal dyeing conditions, the compact structure of polyethylene terphethalate fibers makes the penetration of disperse dyes inside them very difficult. Dyeing of polyethylene terephthalate fibers therefore requires special conditions such as high temperature $\left(\sim 130^{\circ} \mathrm{C}\right)$, dry heat $\left(190-220^{\circ} \mathrm{C}\right)$, or using carrier in the dye bath $[10,11,12,13]$.

The chemical structure of disperse dyes contains polar groups such as $-\mathrm{NHR},-\mathrm{OH}$ and $\mathrm{NH}_{2}$ but there are no ionic groups present which leads to their very low solubility in water $[14,15]$. Azo, anthraquinone, and nitro diphenylamine constitute the three main chemical structure of disperse dyes. However, as far as the application is concerned, these dyes are divided into four groups namely A, B, C, and D [15]. Temperature, time, and disperse dye concentration are the primary factors affecting the color yield in dyeing polyethylene terephthalate. The overall picture of the relative importance of these factors can be seen in models representing the color yield as a function of them. These models may also have applications in processing and cost minimization [10].

Our objective is to present a model for the color yield of polyethylene terephthalate dyed with specific disperse dyes by high temperature method. The model will represent color yield as a function of time, temperature and dye concentration for each dye. $K / S$ has a direct relationship with the color yield. $K / S$ shows the ratio of absorbed light by an opaque substrate relative to the scattered light from it. This ratio is calculated by Kubelka-Munk theory as follows:

$$
(K / S)_{\lambda}=\frac{\left(1-R_{\lambda}\right)^{2}}{2 R_{\lambda}},
$$

where $R_{\lambda}$ is the reflectance of sample of infinite thichness to light of given wavelength, expressed in fractional form [16].

\section{Modeling of Polyester Dyeing}

As mentioned before, the present study aims to model variations of color yield of polyester samples dyed with different disperse dyes versus time, temperature, and disperse dye concentration in the high temperature (HT) polyester dyeing process. To this end, Mamdani systems as well as fuzzy pattern trees are used. 


\begin{tabular}{ll}
\hline Index Name & Chromophore \\
\hline C.I. Disperse Blue 266 & Mono Azo \\
C.I. Disperse Brown 1 & \\
C.I. Disperse Blue 56 & Anthraquinone \\
C.I. Disperse Red 60 & \\
C.I. Disperse Yellow 7 & Diazo \\
C.I. Disperse Yellow 23 & \\
\hline
\end{tabular}

TABLE I: THE DISPERSE DYES EMPLOYED FOR SAMPLES DYEING.

Dyeing of the samples ( $5 \mathrm{~g}$ ) was carried out by Polymat laboratory dyeing machine (AHIBA 1000) with the following recipe:

$\begin{array}{lc}\text { Disperse dye } & \mathrm{x} \% \\ \mathrm{pH} & 5.5 \\ \mathrm{~L}: \mathrm{R} & 50: 1\end{array}$

and by systematically varying the parameters as follows [17]:

$$
\begin{aligned}
\text { dye concentration } & \in\{0.75,1.5,3,4.5,6\} \\
\text { dyeing temperature } & \in\{100,110,115,120,125,130\} \\
\text { dyeing time } & \in\{12,24,36,48\}
\end{aligned}
$$

After dyeing, reduction clearing for the samples was carried out for 10 minutes in a bath $\left(65^{\circ} \mathrm{C}\right)$ containing sodium hydroxide $38^{\circ} \mathrm{Be}$, sodium dithionite and a nonionic detergent $[10,17]$. The disperse dyes employed are listed in Table $\mathrm{i}$ $[10,17]$.

From the values for concentration, temperature and time, 120 combinations were constructed, for which the output K/S was determined experimentally. Thus, for a single disperse dye, a data set with 120 observations was obtained (hence 6 such data sets in total), where each observations consists of three values of the input attributes (concentration, temperature, time) and one value for the output (K/S).

In the following, we provide an overview of the different approaches to fuzzy systems modeling that will be compared in Section 5.

\subsection{Mamdani Systems}

Classical Mamdani inference systems [18] are often considered as most convenient from a modeling perspective. The Mamdani model for representing the color yield of C.I. Disperse Blue 266 as a function of time, temperature, and disperse dye concentration in the high temperature (HT) polyester dyeing, was developed in a purely knowledge-driven way. To this end, expert knowledge was formalized as follows [19]: First, membership functions for input and output variables regarding HT dyeing of polyester for C.I. Disperse Blue 266, one of mono Azo Disperse Dyes, have been determined. Gaussian membership functions were used for all input and output variables. The values for mean and spread of membership functions for each variable are given in Tables ii and iii.

In the second stage, the eight rules blow were defined according to the physical and chemical structure of polyester fiber, HT dyeing of polyester, and the behavior of 120 samples dyed in C.I. Disperse Blue 266 [19]:

1. If (temperature is low) and (time is low) and (concentration is low), then (K/S is very low).

\begin{tabular}{lcccccc}
\hline & \multicolumn{2}{c}{ Concentration } & \multicolumn{2}{c}{ Time } & \multicolumn{2}{c}{ Temperature } \\
Fuzzy Set & Mean & Std & Mean & Std & Mean & Std \\
\hline Low & 0.30 & 1.00 & 13.1 & 14.2 & 100 & 7.8 \\
Medium & 3.38 & 0.89 & - & - & 117 & 3.0 \\
High & 5.86 & 1.33 & 44.4 & 11.7 & 129 & 4.0 \\
\hline
\end{tabular}

TABLE II: PARAMETERS OF GAUSSIAN FUZZY SETS FOR INPUT VARIABLES.

\begin{tabular}{lcc}
\hline Fuzzy Set & mean & spread \\
\hline Very Low & 0.00 & 3.30 \\
Low & 4.11 & 1.42 \\
Medium & 12.8 & 1.69 \\
High & 19.3 & 1.86 \\
Very High & 29.80 & 3.50 \\
\hline
\end{tabular}

TABLE III: PARAMETERS OF GAUSSIAN FUZZY SETS FOR OUTPUT VARIABLE.

2. If (temperature is medium) and (concentration is high), then (K/S is high).

3. If (temperature is high) and (concentration is low), then ( $\mathrm{K} / \mathrm{S}$ is medium).

4. If (temperature is low) and (time is high) and (concentration is low), then (K/S is very low).

5. If (temperature is high) and (concentration is high), then ( $\mathrm{K} / \mathrm{S}$ is very high).

6. If (temperature is medium) and (time is low) and (concentration is high), then (K/S is medium).

7. If (temperature is medium) and (time is high) and (concentration is high), then (K/S is high).

8. If (Temperature is low) and (time is low) and (concentration is high), then $\mathrm{K} / \mathrm{S}$ is low.

In a manner similar to the one described above, the proposed method was applied to C.I. Disperse Brown 1, which has a chemical structure similar to C.I. Disperse Blue 266, with no changes in the FIS designed for the previous dye. The obtained FIS has also been applied for Anthraquinone dyes.

Regarding the different chemical structure of Diazo dyes, the behavior of each of them has been studied and the parameters of temperature according to change of color yield in each of them during change of temperature have been defined as shown in Table iv.

\subsection{Fuzzy Pattern Trees}

Fuzzy pattern trees have been designed in three different ways: purely knowledge-driven, purely data-driven, and using a hybrid approach combining knowledge-driven and datadriven modeling.

\begin{tabular}{lcc}
\hline & \multicolumn{2}{c}{ Diazo } \\
Fuzzy Set & mean & spread \\
\hline Low & 100.00 & 5.00 \\
Medium & 115.00 & 5.00 \\
High & 130.00 & 5.00 \\
\hline
\end{tabular}

TABLE IV: PARAMETERS OF FUZZY SETS FOR VARIABLE 'TEMPERATURE' FOR DIAZO DYES. 


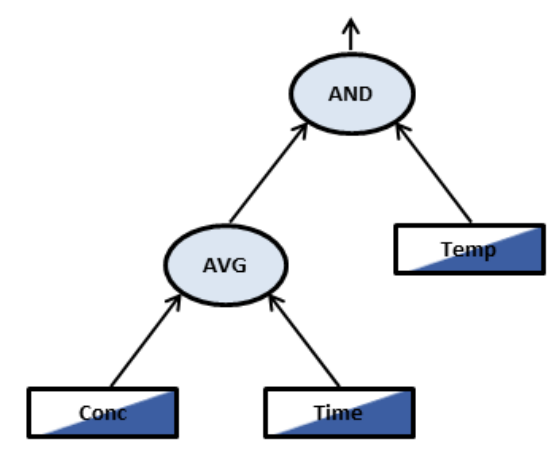

Fig. 2: Knowledge-driven fuzzy pattern trees for Mono Azo and Anthraquinone dyes.

\subsubsection{Knowledge-Driven Modeling}

The fuzzy pattern trees defined by the expert is shown in Figure 2. This model, which applies to Mono Azo and Anthraquinone disperse dyes, is derived from the consideration that temperature plays the most important role for the corresponding chemical groups; therefore the property of having a "high temperature" is specified as the first main criterion to guarantee a high color yield. Since a high concentration is important, too, the temperature criterion is combined conjunctively (using the minimum operator) with a second criterion, which combines concentration and dyeing time by a weighted mean. Since the former is considered as being more important than the latter, the property of having a larger weight for concentration and a smaller one for time is specified as an additional constraint by the expert.

The definition of the FPT model for Diazo dyes follows a similar line of reasoning. However, since a high concentration is now considered as the most important criterion, concentration and temperature change their role.

The fuzzy sets "high" that are needed for all three parameters have been defined in terms of membership functions of piecewise linear the form

$$
x \mapsto \max \left(\min \left(\frac{x-a}{b-a}, 1\right), 0\right) .
$$

The two parameters $a$ and $b$ are given by the corresponding minimum and maximum values in the parameters ranges (1).

\subsubsection{Data-Driven Modeling}

The purely data-driven approach to fuzzy pattern tree modeling, subsequently be denoted by FPT-DD, has been realized by means of the learning algorithm proposed in [7].

\subsubsection{Hybrid Modeling}

We considered two hybrid variants of pattern tree construction, in which expert knowledge is incorporated in the learning process. In the first variant, the fuzzy sets "high" are predefined by the expert for each parameter. Apart from that, the model is learned as ususal, i.e., the tree structure as well as the aggregation functions and their parameters are determined in a data-driven way. This variant will subsequently be denoted by FPT-DE.
The second approach is more knowledge-driven. Here, a complete FPT is specified by the expert, including the structure and the type of aggregation functions. Only the parameters of this model (i.e., the parameters of the aggregation functions and the parameters of the underlying fuzzy sets (2)) are calibrated in a data-driven way. To this end, the calibration procedure proposed in [20] has been used, which is based on evolutionary optimization techniques. We shall refer to this variant as FPT-EC.

\section{EXPerimental Comparison}

Experimentally, we compared the different variants of pattern tree induction (FPT-DD, FPT-DE, FPT-EC) with the purely knowledge-driven approach based on Mamdani models (Exp). As additional baselines, we furthermore included standard linear regression (LR) and quadratic regression (QR).

The accuracy of the models has been determined by training them on a randomly selected sample of $K \in$ $\{20,40,60,80,100\}$ instances and evaluating (in terms of root mean squared error, RMSE) on the remaining $120-K$ samples; we report average and standard deviation of the RMSE over 200 repetitions of this procedure. The results for the different values of $K$ are summarized in Tables v-vii; moreover, the mean accuracies are shown graphically for the different colors in Figure 3. Finally, Table viii shows the size of the FPT models in terms of the number of nodes of the trees. These results support the following conclusions:

- The regression models are not competitive in terms of accuracy, apparently since they are not able to adequately capture the nonlinear dependencies in the data.

- The FPT models perform best, whereas the knowledgedriven Mamdani models are in-between.

- Perhaps most importantly, consistent and significant improvements can be achieved by the hybrid variants. The hybrid models are not only smaller in size but also superior in terms of generalization performance.

- Compared to Mamdani models, the hybrid FPT models are not only more accurate but also found to be more interpretable, especially since they are small and very compact.

- The (hybrid) FPT models stabilize their performance quite quickly, i.e., relatively few training examples are enough to reach an (almost) optimal performance; this is not surprising, given that small trees do not have many parameters (degrees of freedom) to be fixed.

\section{Conclusions}

We interpret our results in favor fuzzy pattern trees as a viable alternative to conventional rule-based fuzzy systems design. In fact, the model class of fuzzy pattern trees is not only interesting from the point of view of data-driven model construction. Instead, it also appears to provide a convenient tool for modeling expert knowledge in a purely knowledge-driven way. Most importantly, however, our study suggests that even better models can be produced by combining expert knowledge with data-driven model construction. Indeed, thanks to our hybrid variants, we obtained models that are more accurate and, at the same time, smaller in size.

Needless to say, the case study presented in this paper is still of limited complexity. In future work, we therefore plan to evaluate our methods on more complex modeling tasks. 

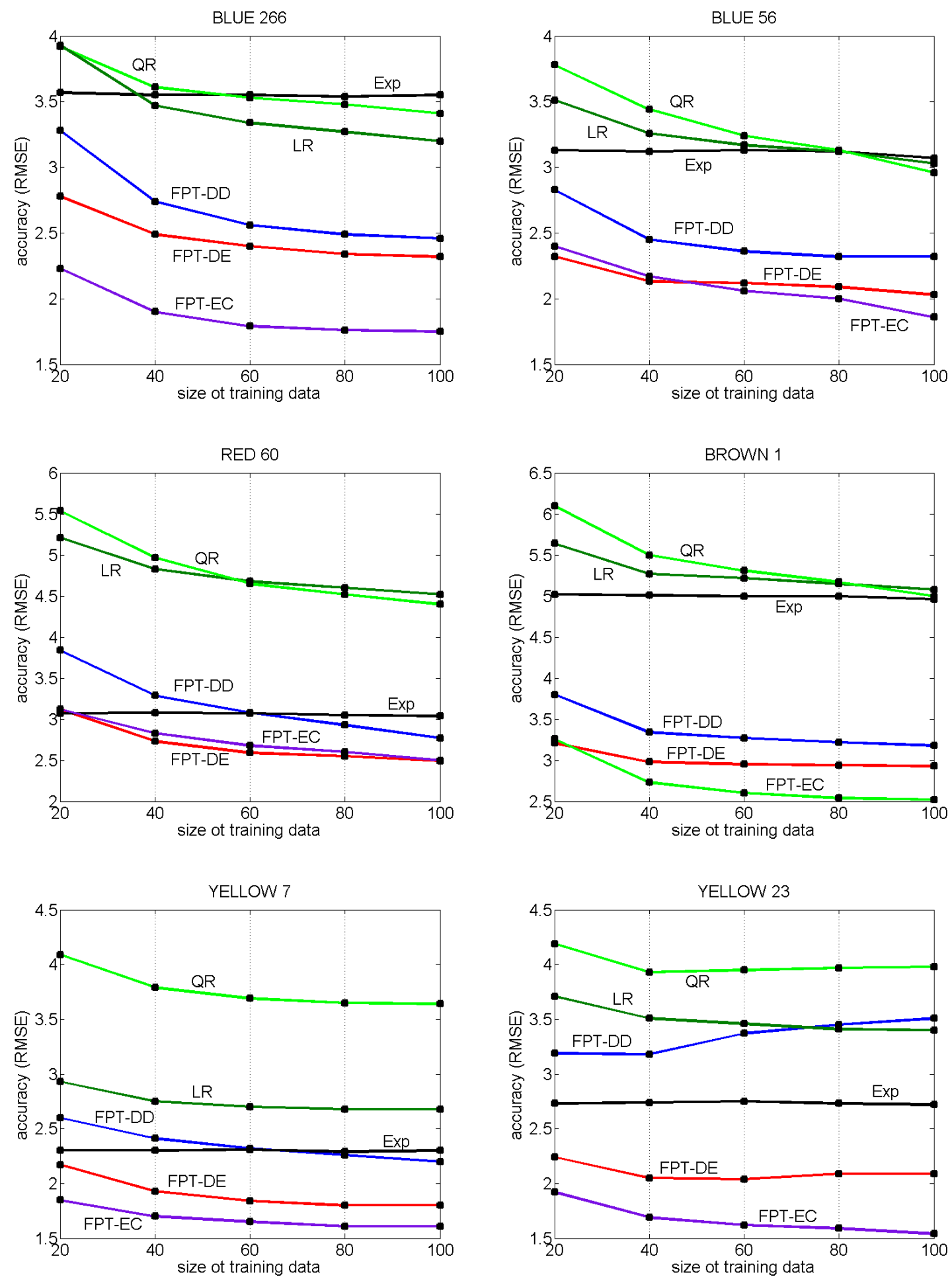

Fig. 3: Generalization accuracy for the different colors as a function of the size of training data. 


\begin{tabular}{lcccccc}
\hline Color & FPT-DD & FPT-DE & Exp & FPT-EC & LR & QR \\
\hline Blue 266 & $3.28 \pm 0.77$ & $2.78 \pm 0.71$ & $3.57 \pm 0.11$ & $2.23 \pm 0.42$ & $3.92 \pm 0.34$ & $3.93 \pm 0.43$ \\
Blue 56 & $2.83 \pm 0.62$ & $2.32 \pm 0.49$ & $3.13 \pm 0.06$ & $2.40 \pm 0.36$ & $3.51 \pm 0.31$ & $3.78 \pm 0.46$ \\
Red 60 & $3.84 \pm 0.86$ & $3.12 \pm 0.71$ & $3.07 \pm 0.12$ & $3.12 \pm 0.42$ & $5.21 \pm 0.54$ & $5.54 \pm 0.65$ \\
Brown 1 & $3.80 \pm 0.95$ & $3.21 \pm 0.59$ & $5.02 \pm 0.14$ & $3.26 \pm 0.75$ & $5.64 \pm 0.63$ & $6.10 \pm 0.77$ \\
Yellow 7 & $2.60 \pm 0.45$ & $2.17 \pm 0.49$ & $2.30 \pm 0.09$ & $1.85 \pm 0.29$ & $2.93 \pm 0.28$ & $4.09 \pm 0.41$ \\
Yellow 23 & $3.19 \pm 0.59$ & $2.24 \pm 0.65$ & $2.73 \pm 0.06$ & $1.92 \pm 0.32$ & $3.71 \pm 0.33$ & $4.19 \pm 0.53$ \\
\hline
\end{tabular}

TABLE V: ACCURACY IN TERMS OF RMSE \pm STANDARD DEVIATION IF MODELS ARE TRAINED ON 20 EXAMPLES.

\begin{tabular}{lcccccc}
\hline Color & FPT-DD & FPT-DE & Exp & FPT-EC & LR & QR \\
\hline Blue 266 & $2.56 \pm 0.29$ & $2.40 \pm 0.26$ & $3.55 \pm 0.25$ & $1.79 \pm 0.16$ & $3.53 \pm 0.25$ & $3.34 \pm 0.24$ \\
Blue 56 & $2.36 \pm 0.24$ & $2.12 \pm 0.31$ & $3.13 \pm 0.17$ & $2.06 \pm 0.26$ & $3.17 \pm 0.26$ & $3.24 \pm 0.36$ \\
Red 60 & $3.08 \pm 0.45$ & $2.59 \pm 0.32$ & $3.07 \pm 0.25$ & $2.68 \pm 0.33$ & $4.68 \pm 0.35$ & $4.65 \pm 0.38$ \\
Brown 1 & $3.27 \pm 0.31$ & $2.95 \pm 0.26$ & $5.00 \pm 0.30$ & $2.60 \pm 0.31$ & $5.22 \pm 0.32$ & $5.31 \pm 0.36$ \\
Yellow 7 & $2.32 \pm 0.33$ & $1.84 \pm 0.15$ & $2.31 \pm 0.20$ & $1.65 \pm 0.17$ & $2.70 \pm 0.19$ & $3.69 \pm 0.21$ \\
Yellow 23 & $3.37 \pm 0.43$ & $2.04 \pm 0.23$ & $2.75 \pm 0.15$ & $1.62 \pm 0.21$ & $3.46 \pm 0.26$ & $3.95 \pm 0.33$ \\
\hline
\end{tabular}

TABLE VI: ACCURACY IN TERMS OF RMSE \pm STANDARD DEVIATION IF MODELS ARE TRAINED ON 60 EXAMPLES.

\begin{tabular}{|c|c|c|c|}
\hline color & $\bar{K}$ & FPT-DD & FPT-DE \\
\hline \multirow[t]{5}{*}{ Blue 266} & 20 & $10.71 \pm 1.29$ & $10.89 \pm 0.78$ \\
\hline & 40 & $10.97 \pm 0.42$ & $10.97 \pm 0.32$ \\
\hline & 60 & $11.00 \pm 0.00$ & $11.00 \pm 0.00$ \\
\hline & 80 & $11.00 \pm 0.00$ & $11.00 \pm 0.00$ \\
\hline & 100 & $11.00 \pm 0.00$ & $11.00 \pm 0.00$ \\
\hline \multirow[t]{5}{*}{ Blue 56} & 20 & $6.08 \pm 3.83$ & $9.53 \pm 2.87$ \\
\hline & 40 & $4.91 \pm 3.23$ & $9.13 \pm 3.34$ \\
\hline & 60 & $4.29 \pm 2.83$ & $8.07 \pm 3.78$ \\
\hline & 80 & $4.22 \pm 2.86$ & $8.04 \pm 3.79$ \\
\hline & 100 & $3.44 \pm 1.73$ & $8.50 \pm 3.65$ \\
\hline \multirow[t]{5}{*}{ Red 60} & 20 & $7.98 \pm 3.81$ & $8.63 \pm 3.26$ \\
\hline & 40 & $6.97 \pm 3.96$ & $9.28 \pm 2.84$ \\
\hline & 60 & $7.63 \pm 3.88$ & $9.53 \pm 2.44$ \\
\hline & 80 & $8.51 \pm 3.71$ & $9.98 \pm 2.01$ \\
\hline & 100 & $9.96 \pm 2.66$ & $10.57 \pm 1.43$ \\
\hline \multirow[t]{5}{*}{ Brown 1} & 20 & $8.87 \pm 3.40$ & $8.63 \pm 3.36$ \\
\hline & 40 & $8.65 \pm 3.41$ & $8.51 \pm 3.54$ \\
\hline & 60 & $9.18 \pm 3.19$ & $8.23 \pm 3.67$ \\
\hline & 80 & $9.83 \pm 2.62$ & $6.67 \pm 3.82$ \\
\hline & 100 & $10.85 \pm 1.06$ & $5.12 \pm 3.36$ \\
\hline \multirow[t]{5}{*}{ Yellow 7} & 20 & $9.97 \pm 2.54$ & $10.40 \pm 1.67$ \\
\hline & 40 & $10.07 \pm 2.51$ & $10.69 \pm 1.08$ \\
\hline & 60 & $10.03 \pm 2.48$ & $10.75 \pm 1.10$ \\
\hline & 80 & $10.49 \pm 1.92$ & $10.97 \pm 0.42$ \\
\hline & 100 & $10.82 \pm 1.03$ & $11.00 \pm 0.00$ \\
\hline \multirow[t]{5}{*}{ Yellow 23} & 20 & $7.63 \pm 3.88$ & $7.52 \pm 3.64$ \\
\hline & 40 & $6.51 \pm 3.90$ & $6.44 \pm 3.81$ \\
\hline & 60 & $4.64 \pm 3.20$ & $6.05 \pm 3.81$ \\
\hline & 80 & $3.64 \pm 2.12$ & $4.21 \pm 2.84$ \\
\hline & 100 & $3.13 \pm 0.98$ & $3.29 \pm 1.39$ \\
\hline
\end{tabular}

TABLE VIII: SIZE OF THE PATTERN TREE MODELS FOR THE SIX DIFFERENT COLORS AND DIFFERENT AMOUNTS OF TRAINING DATA.
Acknowledgments: This work was partially funded by the German Research Foundation (DFG). The first author likes to thank Prof. Hossein Tavanai for his important assistance in the data acquisition process, during the work on her M.Sc. dissertation.

\section{REFERENCES}

[1] J. Casillas, O. Cordon, F. Herrera, and L. Magdalena, editors. Interpretability Issues in Fuzzy Modeling. Studies in Fuzziness and Soft Computing. Springer-Verlag, Berlin, 2003.

[2] H. Ishibuchi and Y. Nojima. Analysis of interpretabilityaccuracy tradeoff of fuzzy systems by multiobjective fuzzy genetics-based machine learning. International Journal of Approximate Reasoning, 44(1):4-31, 2007.

[3] J.M. Alonso and L. Magdalena. A conceptual framework for understanding a fuzzy system. In Proc. IFSA/EUSFLAT-2009, pages 119-124, Lisbon, Portugal, 2009.

[4] J.M. Alonso and L. Magdalena. An experimental study on the interpretability of fuzzy systems. In Proc. IFSA/EUSFLAT2009, pages 125-130, Lisbon, Portugal, 2009.

[5] M. Nasiri, E. Hüllermeier, R. Senge, and E. Lughofer. Comparing methods for knowledge-driven and data-driven fuzzy modeling: A case study in textile industry. In Proceedings IFSA2011, World Congress of the International Fuzzy Systems Association, pages RW-103-1-6, Surabaya and Bali Island, Indonesia, 2011.

[6] Z. Huang, T.D. Gedeon, and M. Nikravesh. Pattern tree induction: A new machine learning method. IEEE Transactions on Fuzzy Systems, 16(4):958-970, 2008.

[7] R. Senge and E. Hüllermeier. Top-down induction of fuzzy pattern trees. IEEE Transactions on Fuzzy Systems, 19(2):241$252,2011$.

[8] R. Senge and E. Hüllermeier. Pattern trees for regression and fuzzy systems modeling. In Proceedings WCCI-2010, World Congress on Computational Intelligence, Barcelona, Spain, 2010.

[9] T. Takagi and M. Sugeno. Fuzzy identification of systems and its applications to modeling and control. IEEE Trans. on Systems, Man and Cybernetics, 15(1):116-132, 1985.

[10] H. Tavanai, S.M. Taheri, and M. Nasiri. Modeling of colour yield in polyethylene terphethalate dyeing with statistical and fuzzy regression. Iranian Polymer Journal, 14(11):954-968, 2005.

[11] J. Goorden Cook. HandBook of Textile Fibers, Man Made Fiber. Merro Technical Library, 1984. 


\begin{tabular}{lcccccc}
\hline Color & FPT-DD & FPT-DE & Exp & FPT-EC & LR & QR \\
\hline Blue 266 & $2.46 \pm 0.39$ & $2.32 \pm 0.39$ & $3.55 \pm 0.55$ & $1.75 \pm 0.28$ & $3.41 \pm 0.48$ & $3.20 \pm 0.43$ \\
Blue 56 & $2.32 \pm 0.39$ & $2.03 \pm 0.52$ & $3.07 \pm 0.38$ & $1.86 \pm 0.49$ & $3.03 \pm 0.48$ & $2.96 \pm 0.45$ \\
Red 60 & $2.77 \pm 0.62$ & $2.49 \pm 0.54$ & $3.04 \pm 0.59$ & $2.50 \pm 0.53$ & $4.52 \pm 0.66$ & $4.40 \pm 0.66$ \\
Brown 1 & $3.18 \pm 0.52$ & $2.93 \pm 0.54$ & $4.96 \pm 0.71$ & $2.52 \pm 0.44$ & $5.08 \pm 0.66$ & $5.00 \pm 0.57$ \\
Yellow 7 & $2.20 \pm 0.36$ & $1.80 \pm 0.28$ & $2.30 \pm 0.46$ & $1.61 \pm 0.30$ & $2.68 \pm 0.39$ & $3.64 \pm 0.40$ \\
Yellow 23 & $3.51 \pm 0.46$ & $2.09 \pm 0.38$ & $2.72 \pm 0.33$ & $1.54 \pm 0.33$ & $3.40 \pm 0.48$ & $3.98 \pm 0.54$ \\
\hline
\end{tabular}

TABLE VII: ACCURACY IN TERMS OF RMSE \pm STANDARD DEVIATION IF MODELS ARE TRAINED ON 100 EXAMPLES

[12] E.R. Trotman. Dyeing and Chemical Technology of Textile Fibers. Charles Griffin, 1970.

[13] R.W. Moncrieff. Man-Made Fibers. Newnes-Butterworths, 1971.

[14] A. Johnon. The Theory of Coloration of Textiles. Bardford: S.D.C. Pub., 2nd edition, 1989.

[15] D.M. Nunn. The Dyeing of Synthetic Polymer and Acetate Fiber. 1979.

[16] W. Baumann et al. Determination of relative colour strength and residual color difference by means of reflectance measurement. J. Soc. Dyers and Colour, 103:100-105, 1987.

[17] M. Nasiri. Fuzzy regression modeling of colour yield in dyeing polyester with disperse dyes. Master's thesis, Textile Engineering Department, Isfahan University of Technology, Iran, 2003.

[18] E. Mamdani and S. Assilian. An experiment in linguistic synthesis with a fuzzy logic controller. International Journal of Man-Machine Studies, 7:1-13, 1975.

[19] M. Nasiri and S. Berlik. Modeling of polyester dyeing using an evolutionary fuzzy system. In Proc. IFSA/EUSFLAT-2009, pages 1246-1251, Lisbon, Portugal, 2009.

[20] Y. Yi, T. Fober, and E. Hüllermeier. Fuzzy operator trees for modeling rating functions. International Journal of Computational Intelligence and Applications, 8(4):413-428, 2009. 